\title{
Finite density 2D $O(3)$ sigma model: Dualization and numerical simulations
}

\author{
B. Allés* \\ INFN Sezione di Pisa, Largo Pontecorvo 3, 56127 Pisa, Italy \\ O. Borisenko ${ }^{\dagger}$ \\ N.N. Bogolyubov Institute for Theoretical Physics, National Academy of Sciences of Ukraine, \\ 03143 Kiev, Ukraine \\ A. Papa \\ Dipartimento di Fisica, Università della Calabria and INFN Gruppo Collegato di Cosenza, \\ Arcavacata di Rende, 87036 Cosenza, Italy
}

(Received 23 August 2018; revised manuscript received 7 November 2018; published 18 December 2018)

\begin{abstract}
The action of the 2D $O(3)$ nonlinear sigma model on the lattice in a bath of particles, when expressed in terms of standard $O(3)$ degrees of freedom, is complex. A reformulation of the model in terms of new variables that makes the action real is presented. This reshaping enables us to utilize Monte Carlo simulations based on usual importance sampling. Several observables, including the correlation function and the mass gap, are measured.
\end{abstract}

DOI: $10.1103 /$ PhysRevD.98.114508

\section{INTRODUCTION}

The physics of dense matter is crucial for understanding the drastic changes undergone by the Universe during the first minutes after the big bang. The extreme conditions that prevailed in that period can be partially reproduced in experiments with heavy-ion collisions where the effects of dense matter are probed.

The presence of a density of matter affects the dynamical laws that govern the physical systems. The study of these effects by numerical simulations is usually hindered by the so-called sign problem. This problem consists in the fact that upon adding a coupling with an external chemical potential, the functional that weighs field configurations may not furnish a positive number, thus ruining any attempt to use that functional as a probability for importance sampling in a Monte Carlo procedure.

Several methods have been proposed to overcome those difficulties in Monte Carlo simulations of quantum chromodynamics (QCD), see for instance Ref. [1]. The quest for better strategies has raised the interest in 2D toy models that are afflicted by similar problems [2-9]. A technique that has proven to be particularly appealing to

\footnotetext{
*alles@pi.infn.it

oleg@bitp.kiev.ua

papa@fis.unical.it
}

Published by the American Physical Society under the terms of the Creative Commons Attribution 4.0 International license. Further distribution of this work must maintain attribution to the author(s) and the published article's title, journal citation, and DOI. Funded by SCOAP ${ }^{3}$. evade the sign problem is dualization: the model is recast in terms of new (dual) variables in such a way that the new action turns out to be real.

No general recipe exists for transforming ordinary into dual variables. Every model has to be studied on its own and the strategy to get a dual representation may differ significantly for different models. Moreover, not even a unique dual representation exists for a given model. In fact, among the few different possible dual representations available for a given model, some may be more advantageous than others for simulation purposes or some might even present a so heavy slowing down that it makes the dual version, albeit real, useless.

Another type of drawback that often appears in dual formulations is that certain observables cannot be disentangled from the probability weight in such a way that their expectation values have to be extracted as the ratio of expectation values with different Hamiltonians, which in general gives rise to extremely inefficient numerical calculations. Such difficulties typically arise in the evaluation of nonlocal observables like correlation functions.

In the present paper we apply the dualization idea to the 2D $O(3)$ nonlinear sigma model in presence of a chemical potential. The standard action of the model on a $d$-dimensional lattice $\Lambda \in \mathbb{Z}^{d}$ without a chemical potential is given by

$$
S=\sum_{x, \nu} \sum_{k=1}^{3} \sigma_{k}(x) \sigma_{k}\left(x+e_{\nu}\right)
$$


together with the condition $\sum_{k} \sigma_{k}^{2}(x)=1$ for every $x$. The corresponding partition function reads

$$
Z_{\Lambda}(\beta)=\int \prod_{x \in \Lambda} \prod_{k=1}^{3} d \sigma_{k}(x) \prod_{x \in \Lambda} \delta\left(1-\sum_{k=1}^{3} \sigma_{k}^{2}(x)\right) \exp [\beta S] .
$$

In 2D this model possesses a particle triplet with a spontaneously generated mass gap $m$ [10]. The value of this mass has been verified numerically $[11,12]$. The model is asymptotically free and presents a rich topology [13]. All those properties make this model a close relative of QCD.

The physical interest of the $2 \mathrm{D} O(3)$ nonlinear sigma model goes well beyond the above list of properties. In the first place the nonlinear sigma model reproduces reliably several qualitative traits of ferromagnetic materials. Another reason for casting relevance to the model is that it is involved in the development of the resurgence program $[14,15]$.

Our purpose is to construct a real action for the 2D $O(3)$ nonlinear sigma model at finite density expressed in terms of dual variables and in such a way that it allows us to determine vacuum expectation values with usual Monte Carlo methods.

To describe the theory at finite density one introduces a chemical potential $\mu$ in the original action as an external source for the total third component of the angular momentum [10]. Following [16] and using the spherical parametrization

$$
\sigma_{1}=\sin \alpha \cos \phi, \quad \sigma_{2}=\sin \alpha \sin \phi, \quad \sigma_{3}=\cos \alpha,
$$

we derive the standard action with a nonzero chemical potential

$$
\begin{aligned}
S(\{\alpha(x), \phi(x)\})= & \sum_{x, \nu}\left[\cos \alpha(x) \cos \alpha\left(x+e_{\nu}\right)\right. \\
& +\sin \alpha(x) \sin \alpha\left(x+e_{\nu}\right) \cos (\phi(x) \\
& \left.\left.-\phi\left(x+e_{\nu}\right)-i \mu_{\nu}\right)\right],
\end{aligned}
$$

where the general situation with anisotropic chemical potentials $\mu_{\nu}, \nu=1,2$ is considered. The conventional physical situation is recovered if we put $\mu_{1}=\mu, \mu_{2}=0$.

Three different routes to introduce dual variables are possible, and it is not obvious a priori which one is preferable and under what circumstances may it be so. The first route relies on the fact that the chemical potential is introduced in the Abelian, i.e., $O(2)$, subgroup. The $O(2)$ part of the general $O(N)$ action has the form

$$
S=\sum_{x, \nu} \beta_{\nu}(x) \cos \left(\phi(x)-\phi\left(x+e_{\nu}\right)-i \mu_{\nu}\right) .
$$

This action is of an $X Y$ type with a fluctuating coupling and in the presence of $\mu_{\nu}$. As shown in [17] in the case of the Villain formulation of the $X Y$ model and for $\beta_{\nu}(x)=\beta$ for all $x$, the conventional dual transformations performed with a nonvanishing chemical potential lead to a positive definite Boltzmann weight. Moreover, if the coupling $\beta_{\nu}(x)$ is positive for all $x$, then it is straightforward to prove that exactly the same transformations lead to a positive dual weight for all $O(N)$ models. This conclusion has been explicitly demonstrated in [18] for the 2D $O(3)$ model, and the proof can be readily extended to all $O(N)$ models.

The second route consists in Taylor expanding the Boltzmann weight and integrating over the original degrees of freedom. The dual variables appear as flux variables subject to certain constraints, and the dual weight can be proven to be positive for $O(N)$ models $[19,20]$. The resulting dual theory can be simulated by a worm algorithm, and a number of thermodynamic quantities have been computed along this route [19-21].

In the third route one constructs the dual theory by expanding the Boltzmann weight in hyperspherical harmonics on $O(N)$ and integrating out the original variables. This program has been accomplished for the 2D $O(3)$ model in [18]. However, the full positivity of the resulting dual weight remains to be proven. It is important to stress that, at least in the context of these two-dimensional models, the dual formulation appears as the only reliable tool to investigate the properties of the model. Indeed, the results of Ref. [21] show that alternative approaches to the sign problem - the reweighting and the complex Langevin - have certain drawbacks and lead to incorrect results in some regions of the $\beta-\mu$ plane.

One of the main purposes of the present work is to develop a dual formulation applicable not only for computing thermodynamic quantities, but also for extracting longdistance quantities like the correlation functions. With such a formulation in hand, one could be able to reliably address the question of the hypothetical Berezinskii-KosterlitzThouless (BKT) transition in $O(N)$ models at nonvanishing chemical potential. Our approach is essentially the first route described above. We reformulate the $O(N)$ model in terms of the link formulation for the Abelian (sub)group. In this formulation our results can be straightforwardly extended to all $O(N)$ models in any dimension. Here, for the sake of simplicity, we consider only the twodimensional $O(3)$ sigma model.

The paper is organized as follows. In the next section we construct the dual formulation. First we introduce the link representation, then we obtain two alternative dual Boltzmann weights, both of which are positive. To study the real effectiveness of the above Boltzmann weights in Monte Carlo simulations, we test one of them by calculating numerically several observables. We are particularly careful to individuate any slowing down during the simulations. In Sec. III we outline the procedure employed 
during the simulations and list the observables that have been studied. Specifically we calculate the particle density to find the expected threshold at $\mu=m$, verify that the mass gap extracted from a correlation function is insensitive to $\mu$, and evaluate the energy as a function of $\beta$ and $\mu$. The results are presented in Sec. IV and some conclusive remarks in Sec. V.

\section{DUAL REPRESENTATION}

The action (4) becomes complex if any of the $\mu_{\nu}$ 's is nonzero. However, an action with a positive Boltzmann weight can be constructed by using, in the spirit of $[19,20]$, a dual representation of the model with the action (4). Nevertheless, our strategy is somewhat different from $[19,20]$ and relies on the use of the so-called link formulation $[22,23]$. In fact, this approach is similar to the one used in $[17,18]$. One of its advantages is that it can be readily extended to any $O(N)$ model, in any number of space dimensions.

\section{A. Link formulation for Abelian subgroup}

To build a dual representation, we start from the following partition function with the action (4),

$$
Z_{\Lambda}\left(\beta, \mu_{\nu}\right)=\int_{0}^{\pi} \prod_{x} \frac{d \alpha(x)}{2} \sin \alpha(x) \int_{0}^{2 \pi} \prod_{x} \frac{d \phi(x)}{2 \pi} \exp [\beta S(\{\alpha(x), \phi(x)\})]
$$

The integration can be done in a number of ways. Here we use the fact that the dependence of (4) on the angles $\phi(x)$ is only through their differences $(U(1)$ variables). This allows to make a change of variables and rewrite the partition function in terms of the link angles $\phi(l) \equiv \phi_{\nu}(x)=\left|\phi(x)-\phi\left(x+e_{\nu}\right)\right|_{\bmod (2 \pi)}$, where links are defined as $l \equiv(x ; \nu)=\left(x_{1}, x_{2} ; \nu\right)$ if $d=2$.

The procedure generates local and global constraints known as Bianchi identities on the link variables [22,23]. The local identity constrains the allowed configurations of $\phi(l)$ on every plaquette $p$ of the lattice and can be embedded into the partition function in the form of a periodic $\delta$-function as

$$
\prod_{p} \sum_{r(p)=-\infty}^{\infty} e^{i r(p) \phi(p)}, \quad \phi(p)=\phi\left(l_{1}\right)+\phi\left(l_{2}\right)-\phi\left(l_{3}\right)-\phi\left(l_{4}\right), \quad l_{i} \in p .
$$

Global identities constrain two holonomies winding through the lattice in periodic directions. They have the form

$$
\sum_{q_{1}=-\infty}^{\infty} \sum_{q_{2}=-\infty}^{\infty} e^{i q_{1} \sum_{x_{1}} \phi_{1}\left(x_{1}, 0\right)+i q_{2} \sum_{x_{2}} \phi_{2}\left(0, x_{2}\right)}
$$

Then, it is easy to prove the following equality in any number of dimensions $d$ :

$$
\int_{0}^{2 \pi} \prod_{x} \frac{d \phi(x)}{2 \pi} e^{S\left(\left\{\phi(x)-\phi\left(x+e_{\nu}\right)-i \mu_{\nu}\right\}\right)}=\int_{0}^{2 \pi} \prod_{l} \frac{d \phi(l)}{2 \pi} e^{S\left(\left\{\phi(l)-i \mu_{\nu}\right\}\right)} \times \prod_{p} \sum_{r(p)=-\infty}^{\infty} e^{i r(p) \phi(p)} \prod_{\nu=1}^{d} \sum_{q_{\nu}=-\infty}^{+\infty} e^{i q_{\nu} \sum_{x_{\nu}} \phi_{\nu}\left(0, \ldots, x_{\nu}, \ldots, 0\right)} .
$$

One should keep in mind that when $d>2$ not all local Bianchi identities are independent. The chief argument in favor of using the link formulation lies in the following fact. The dependence of the partition function on the chemical potential in the finite temperature theory can appear only through loops winding through the whole lattice in the compactified direction, i.e., through Polyakov loops in terms of gauge theories. In models with a global symmetry group such loops are represented by holonomies which enter in the global Bianchi identities. Therefore, a dependence on the chemical potential can only appear due to nonzero contributions from global variables $q_{\nu}$ representing constraints on such holonomies. This is what the formula (9) demonstrates. Indeed, making a global shift of link variables $\phi(l) \equiv \phi_{\nu}(x) \rightarrow$ $\phi(l)+i \mu_{\nu}$ and using the periodicity of the integrand in (9), one sees that the chemical potential decouples from the integrand and appears in the partition function only through global variables $q_{\nu}$ as $e^{-q_{\nu} \mu_{\nu} L_{\nu}}$. Moreover, this simple transformation brings the partition function to the form in which the contribution of the chemical potential is always real and positive.

Applying this approach to the 2D $O(3)$ model one gets, after integration over link variables, 


$$
\begin{aligned}
Z_{\Lambda}\left(\beta, \mu_{\nu}\right)= & \sum_{q_{1}=-\infty}^{\infty} \sum_{q_{2}=-\infty}^{\infty} e^{-\sum_{\nu=1,2} q_{\nu} \mu_{\nu} L_{\nu}} \sum_{\{r(p)\}=-\infty}^{\infty} \int_{0}^{\pi} \prod_{x} \frac{d \alpha(x)}{2} \sin \alpha(x) \\
& \times \exp \left[\beta \sum_{x, \nu} \cos \alpha(x) \cos \alpha\left(x+e_{\nu}\right)\right] \prod_{x, \nu} I_{r(l)}\left(\beta \sin \alpha(x) \sin \alpha\left(x+e_{\nu}\right)\right),
\end{aligned}
$$

where $L_{\nu}$ are the values of the lattice size in the two directions, $I_{r(l)}$ is the modified Bessel function of first kind, and

$$
r(l)= \begin{cases}r\left(p_{1}\right)-r\left(p_{2}\right)+q_{\nu}, & \text { if } l=\left(x_{1}, 0 ; 1\right) \quad \text { or } \quad l=\left(0, x_{2} ; 2\right) \\ r\left(p_{1}\right)-r\left(p_{2}\right), & \text { otherwise }\end{cases}
$$

The plaquettes $p_{1}$ and $p_{2}$ have the link $l=(x ; \nu)$ in common.

The two-point correlation function between the origin (denoted by a nought 0 ) and a point $R$ in the parametrization (3) reads

$$
\Gamma(R)=\Gamma_{1}(R)+\Gamma_{2}(R)
$$

with

$$
\begin{aligned}
& \Gamma_{1}(R) \equiv\langle\cos \alpha(0) \cos \alpha(R)\rangle, \\
& \Gamma_{2}(R) \equiv\langle\sin \alpha(0) \sin \alpha(R) \cos (\phi(0)-\phi(R))\rangle,
\end{aligned}
$$

where the expectation values $\langle\cdots\rangle$ are evaluated with (6). In the link formulation $\Gamma_{2}(R)$ is given by

$\Gamma_{2}(R)=\left\langle\sin \alpha(0) \sin \alpha(R) \cos \left(\sum_{l \in C_{R}} \eta(l) \phi(l)\right)\right\rangle$, where $\eta(l)$ is defined just below and $C_{R}$ is any lattice path connecting the points 0 and $R$. Introducing a set of sources $\zeta=\left\{h_{1}(x), h_{2}(x), \eta(l)\right\}$ and integrating out link variables one gets

$$
\Gamma_{1}(R)=\frac{Z_{\Lambda}\left(\beta, \mu_{\nu} ; \zeta\right)}{Z_{\Lambda}\left(\beta, \mu_{\nu} ; 0\right)}, \quad \zeta=\left(h_{1}(x), 0,0\right)
$$

where $h_{1}(x)=1$ for $x=0, R$ and $h_{1}(x)=0$ otherwise and

$$
\Gamma_{2}(R)=\frac{1}{2} \frac{Z_{\Lambda}\left(\beta, \mu_{\nu} ; \zeta\right)}{Z_{\Lambda}\left(\beta, \mu_{\nu} ; 0\right)}+\frac{1}{2} \frac{Z_{\Lambda}\left(\beta, \mu_{\nu} ; \zeta^{\prime}\right)}{Z_{\Lambda}\left(\beta, \mu_{\nu} ; 0\right)} .
$$

We have introduced here notations $\zeta=\left(0, h_{2}(x), \eta(l)\right)$ and $\zeta^{\prime}=\left(0, h_{2}(x),-\eta(l)\right)$, where $h_{2}(x)=1$ for $x=0, R$ and $h_{2}(x)=0$ otherwise; $\eta(l)=1$ if $l=(x ; \nu) \in C_{R}, \eta(l)=$ -1 if $l=\left(x-e_{\nu} ; \nu\right) \in C_{R}$ and $\eta(l)=0$, otherwise. The partition function utilized in (15) and (16) is given by

$$
\begin{aligned}
Z_{\Lambda}\left(\beta, \mu_{\nu} ; \zeta\right)= & \sum_{q_{1}=-\infty}^{\infty} \sum_{q_{2}=-\infty}^{\infty} e^{-\sum_{\nu=1,2} q_{\nu} \mu_{\nu} L_{\nu}-\sum_{l \in C_{R}} \mu_{\nu} \eta(l)} \times \sum_{\{r(p)\}=-\infty}^{\infty} \int_{0}^{\pi} \prod_{x} \frac{d \alpha(x)}{2} \sin \alpha(x) \exp \left[\beta \sum_{x, \nu} \cos \alpha(x) \cos \alpha\left(x+e_{\nu}\right)\right] \\
& \times \prod_{x}(\cos \alpha(x))^{h_{1}(x)}(\sin \alpha(x))^{h_{2}(x)} \prod_{l} B_{\eta}(l)
\end{aligned}
$$

with

$$
B_{\eta}(l)=I_{r(l)+\eta(l)}\left(\beta \sin \alpha(x) \sin \alpha\left(x+e_{\nu}\right)\right) .
$$

As it stands, the expression (17) is much more general and allows to compute correlations of any kind as

$$
\left\langle\prod_{x}(\cos \alpha(x))^{h_{1}(x)}(\sin \alpha(x))^{h_{2}(x)} \prod_{l} \frac{B_{\eta}(l)}{B_{0}(l)}\right\rangle .
$$

\section{B. Dual Boltzmann weight 1}

The dual Boltzmann weight can be read off either from (10) or from (17). It has the form

$$
e^{-\sum_{\nu=1,2} q_{\nu} \mu_{\nu} L_{\nu}} \exp \left[\beta \cos \alpha(x) \cos \alpha\left(x+e_{\nu}\right)+\log B_{0}(l)\right] .
$$

Clearly, it is strictly positive in the integration domain over $\alpha(x)$ and hence it can be used for the numerical simulations. In this case we have

$$
\Gamma_{1}(R)=\langle\cos \alpha(0) \cos \alpha(R)\rangle,
$$




$$
\begin{aligned}
\Gamma_{2}(R)= & \frac{1}{2} e^{-\sum_{l \in C_{R}} \mu_{\nu} \eta(l)}\left\langle\sin \alpha(0) \sin \alpha(R) \prod_{l \in C_{R}} \frac{B_{\eta}(l)}{B_{0}(l)}\right\rangle \\
& +\frac{1}{2} e^{\sum_{l \in C_{R}} \mu_{\nu} \eta(l)}\left\langle\sin \alpha(0) \sin \alpha(R) \prod_{l \in C_{R}} \frac{B_{-\eta}(l)}{B_{0}(l)}\right\rangle .
\end{aligned}
$$

\section{Dual Boltzmann weight 2}

A different dual Boltzmann weight can been constructed by performing a complete integration over the remaining original degrees of freedom.
We begin with the formula

$\int_{0}^{\pi} d \alpha F(\cos \alpha, \sin \alpha)=\sum_{s= \pm 1} \int_{0}^{\pi / 2} d \alpha F(s \cos \alpha, \sin \alpha)$.

This introduces an Ising-like partition function with fluctuating coupling

$$
J_{\nu}(x)=\cos \alpha(x) \cos \alpha\left(x+e_{\nu}\right), \quad \alpha(x) \in[0, \pi / 2] .
$$

Equation (17) becomes

$$
\begin{aligned}
Z_{\Lambda}\left(\beta, \mu_{\nu} ; \zeta\right)= & \sum_{q_{1}=-\infty}^{\infty} \sum_{q_{2}=-\infty}^{\infty} e^{-\sum_{\nu=1,2} q_{\nu} \mu_{\nu} L_{\nu}-\sum_{l \in C_{R}} \mu_{\nu} \eta(l)} \\
& \times \sum_{\{r(p)\}=-\infty}^{\infty} \int_{0}^{\pi / 2} \prod_{x} \frac{d \alpha(x)}{2} \sin \alpha(x) \prod_{x}(\cos \alpha(x))^{h_{1}(x)}(\sin \alpha(x))^{h_{2}(x)} \\
& \times \prod_{l} B_{\eta}(l) Z_{I}\left(\left\{J_{\nu}(x)\right\}\right)
\end{aligned}
$$

where (taking into account that $h_{1}(x)=1$ for $x=0, R$ and zero otherwise)

$$
Z_{I}\left(\left\{J_{\nu}(x)\right\}\right)=\sum_{\{s(x)\}= \pm 1} s(0) s(R) \exp \left[\beta \sum_{x, \nu} J_{\nu}(x) s(x) s\left(x+e_{\nu}\right)\right] .
$$

The dual transformation can be performed in a standard way by introducing the link variables $z(l)=s(x) s\left(x+e_{\nu}\right)$ (this time the global constraints on holonomies can be omitted) to obtain

$$
Z_{I}\left(\left\{J_{\nu}(x)\right\}\right)=\sum_{\{z(l)\}= \pm 1} \prod_{l=1}^{R} z(l) \exp \left[\beta \sum_{x, \nu} J_{\nu}(x) z(l)\right] \prod_{p}\left[\sum_{s(p)=0,1} z(p)^{s(p)}\right],
$$

where $z(p)=\prod_{l \in p} z(l)$. Summation over $z(l)$ leads to the following representation on the dual lattice (now $C_{R}$ is a path connecting the points 0 and $R$ and consisting of links dual to the original links):

$$
Z_{I}\left(\left\{J_{\nu}(x)\right\}\right)=\sum_{\{s(x)\}= \pm 1} \prod_{l \in C_{R}}\left[e^{\beta J_{\nu}(x)}-s(x) s\left(x+e_{\nu}\right) e^{-\beta J_{\nu}(x)}\right] \times \prod_{l \notin C_{R}}\left[e^{\beta J_{\nu}(x)}+s(x) s\left(x+e_{\nu}\right) e^{-\beta J_{\nu}(x)}\right] .
$$

By inserting (28) into (25), we get the expression

$$
\begin{aligned}
Z_{\Lambda}\left(\beta, \mu_{\nu} ; \zeta\right)= & \sum_{q_{1}=-\infty}^{\infty} \sum_{q_{2}=-\infty}^{\infty} e^{-\sum_{\nu=1,2} q_{\nu} \mu_{\nu} L_{\nu}-\sum_{l \in C_{R}} \mu_{\nu} \eta(l)} \sum_{\{r(x)\}=-\infty}^{\infty} \sum_{\{s(x)\}= \pm 1} \\
& \times \int_{0}^{\pi / 2} \prod_{p} \frac{d \alpha(p)}{2}(\cos \alpha(p))^{h_{1}(p)}(\sin \alpha(p))^{h_{2}(p)+1} \prod_{l} B_{\eta}(l) \\
& \times \prod_{l \in C_{R}}\left[e^{\beta J_{\nu}(x)}-s(x) s\left(x+e_{\nu}\right) e^{-\beta J_{\nu}(x)}\right] \prod_{l \notin C_{R}}\left[e^{\beta J_{\nu}(x)}+s(x) s\left(x+e_{\nu}\right) e^{-\beta J_{\nu}(x)}\right] .
\end{aligned}
$$

Here

$$
B_{\eta}(l)=I_{r(l)+\eta(l)}\left(\beta \sin \alpha(p) \sin \alpha\left(p^{\prime}\right)\right),
$$


where

$$
r(l)= \begin{cases}r(x)-r\left(x+e_{\nu}\right)+q_{\nu}, & \text { if } l=\left(x_{1}, 0 ; 2\right) \\ r(x)-r\left(x+e_{\nu}\right), & \text { or } \quad l=\left(0, x_{2} ; 1\right),\end{cases}
$$

The product $\prod_{p}$ in (29) runs over all plaquettes of the dual lattice (in 2D plaquettes are dual to sites and vice-versa). Therefore, the definition of $r(l)$ in (11) takes the form of (31) on the dual lattice. The dual plaquettes $p$ and $p^{\prime}$ have the dual link $l$ in common. Finally, one can integrate out the $\alpha(p)$ angles. This can be done by Taylor expanding the factor $e^{ \pm \beta J_{\nu}(x)}$ and by using either the series representation for the Bessel function or the multiplication theorem for the Bessel function to decouple the $\sin \alpha(p)$ factors from their argument. The first approach is somewhat simpler and leads to the following result:

$$
\begin{aligned}
Z_{\Lambda}\left(\beta, \mu_{\nu} ; \zeta\right)= & \sum_{q_{1}, q_{2}=-\infty}^{\infty} e^{-\sum_{\nu=1,2} q_{\nu} \mu_{\nu} L_{\nu}} \sum_{\{r(x)\}=-\infty}^{\infty} \sum_{\{s(x)\}= \pm 1} \sum_{\{m(l), n(l)\}=0}^{\infty} \\
& \times \prod_{l} \frac{\beta^{n(l)}}{n(l) !} \frac{\left(\frac{\beta}{2}\right)^{2 m(l)+|r(l)+\eta(l)|} e^{-\mu_{\nu} \eta(l)}}{m(l) !(m(l)+|r(l)+\eta(l)|) !} \prod_{p} B\left(\frac{a(p)+1}{2}, \frac{b(p)+1}{2}\right) \\
& \times \prod_{l \in C_{R}}\left[1-s(x) s\left(x+e_{\nu}\right)(-1)^{n(l)}\right] \prod_{l \notin C_{R}}\left[1+s(x) s\left(x+e_{\nu}\right)(-1)^{n(l)}\right],
\end{aligned}
$$

where $B(x, y)$ is the beta-function and $a(p)$ and $b(p)$ are given by

$$
\begin{aligned}
& a(p)=\sum_{l \in p} n(l)+h_{1}(p), \\
& b(p)=1+\sum_{l \in p}(2 m(l)+|r(l)+\eta(l)|)+h_{2}(p) .
\end{aligned}
$$

The partition function can be obtained from the last expression if we put $h_{1}(p)=h_{2}(p)=\eta(l)=0$ and extend the second product in the last line to all links of the lattice

$$
\begin{aligned}
Z_{\Lambda}\left(\beta, \mu_{\nu}\right)= & \sum_{q_{1}, q_{2}=-\infty}^{\infty} e^{-\sum_{\nu=1,2} q_{\nu} \mu_{\nu} L_{\nu}} \sum_{\{r(x)\}=-\infty}^{\infty} \sum_{\{s(x)\}= \pm 1} \sum_{\{m(l), n(l)\}=0}^{\infty} \\
& \times \prod_{l} \frac{\beta^{n(l)}}{n(l) !} \frac{\left(\frac{\beta}{2}\right)^{2 m(l)+|r(l)|}}{m(l) !(m(l)+|r(l)|) !} \prod_{p} B\left(\frac{a(p)+1}{2}, \frac{b(p)+1}{2}\right) \times \prod_{l}\left[1+s(x) s\left(x+e_{\nu}\right)(-1)^{n(l)}\right] .
\end{aligned}
$$

Here, $r(l)$ is given in (31) and

$$
\begin{aligned}
& a(p)=\sum_{l \in p} n(l), \\
& b(p)=1+\sum_{l \in p}(2 m(l)+|r(l)|) .
\end{aligned}
$$

The Boltzmann weights of all three representations (10), (29) and (34) are positive and all interactions between dual variables are local. Moreover, the dual weight of (10) is free of constraints. It means, in particular, that with the help of (10) one can compute not only local quantities (those which can be represented as derivatives of the partition function), but also long-distance quantities like the two-point correlation function, because it can be represented as an ordinary expectation value.

\section{SIMULATION DETAILS}

We have derived two different real Boltzmann weights, (10) and (34), for the same model. However, being real is not the only condition that an action must satisfy to be handy during numerical simulations. In conjunction with an adequate simulation algorithm, the action should also avoid slowing down. Therefore, and in order to elucidate the efficiency of the two weights, we have tested both (10) and (34). The results of physical magnitudes will be presented in the next section but we anticipate that both weights exhibit similar acceptances and simulation efficiencies. The only difference that is worth stressing regards the computation of correlation functions: it is much more problematical with (34) than with (10) because, as is evident from expressions (32) and (34), the correlation function must be determined as the ratio of both quantities 


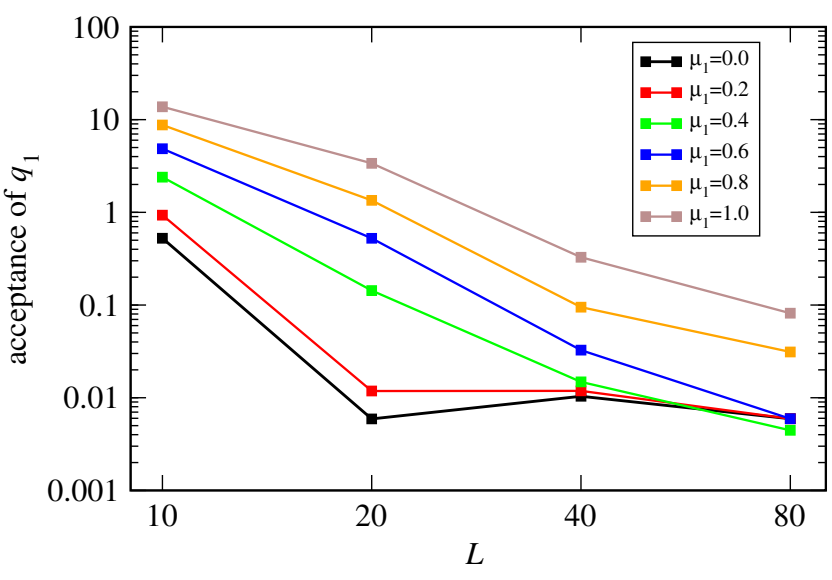

FIG. 1. Acceptance rates of variable $q_{1}$ for $\beta=1.2$ at $\mu_{2}=0$ and for the indicated values of $\mu_{1}$ and of the lattice size. The lines are guides to the eye.

and such ratios are usually so noisy that it is computationally very expensive to prevent the error bars from growing excessively. For all of that, once we verified that the performances of the two weights are equivalent in every respect, we have employed only (10) in the battery of Monte Carlo simulations aimed at extracting physical properties of the model.

We have simulated the weight (10) on square lattices $\Lambda \in \mathbb{Z}^{2}$ of lateral extent ${ }^{1} L_{1}=L_{2} \equiv L$ with periodic boundary conditions. The variables are $\alpha(x) \in[0, \pi]$, $r(p) \in \mathbb{Z}$ and $q_{1}, q_{2} \in \mathbb{Z}$ where $x$ indicates sites and $p$ plaquettes. A single Monte Carlo sweep consists in updating every variable $\alpha(x), r(p)$ and $q_{1}, q_{2}$ with the Metropolis algorithm [24] once.

The refreshing of the angle variables $\alpha(x)$ was done by proposing a brand new value of $\cos \alpha(x)$ with equal probability from the interval $[-1,+1]$, and applying the usual Metropolis test on it. Plaquette variables $r(p)$ and global variables $q_{1}, q_{2}$ were updated after randomly choosing a new value that differs from the old one by at most $\pm \Delta$ units (we took $\Delta=3$ in $r(p)$ and $q_{1}, q_{2}$ ).

The acceptance rate of a dynamical variable is defined as the percentage of these variables that are changed on average during the Monte Carlo sweeps. These rates were generally quite low, particularly for $q_{\mu}$. After setting $\mu_{2}=0$, we show in Fig. 1 the acceptances for $q_{1}$ as a function of $\mu_{1}$ and of the lattice size $L$ for $\beta=1.2$. They exhibit a downward trend as $L$ or $1 / \mu_{1}$ grow, possibly following a power law behavior. Such a behavior will provoke a sudden growth of the error bars for any observable as $L$ and $1 / \mu_{1}$ increase beyond certain values.

The computational times needed to gather $2 \times 10^{7}$ measurements on a $20^{2}$ lattice is about 30 hours on a node with four processors of the type AMD Opteron(tm) Processor 6376.

\footnotetext{
${ }^{1}$ Whenever we write $L$, we will mean that $L_{1}=L_{2} \equiv L$.
}

Next we introduce the physical observables measured in this work. In order to enhance decorrelation, single measurements were evaluated on configurations separated by 10 Monte Carlo sweeps. Error bars were assessed by the Jackknife method applied on blocks of data for further reducing any correlation among raw data. We typically considered ten levels of blocking, the number of blocks ranging from a minimum of ten to a maximum of a few thousands.

The measured magnitudes are

(i) the correlation length which was measured by analysing the wall-wall correlation function. This function was constructed according to the definition (22). Since the path followed to join the walls is irrelevant (on average), we chose it as the simplest one guaranteeing computing efficiency: along the $x_{1}$-axis. For clarity, in Fig. 2 the 16 paths composing a wall-wall correlation at distance 2 from site $(2,0)$ to site $(4,0)$ on a $4 \times 4$ lattice are shown. The total wallwall correlation function at distance 2 consists in summing the contributions from all the above paths and averaging the result over all possible ways the walls can be placed at distance 2: erecting the walls at sites $(1,0)$ and $(3,0)$, at $(2,0)$ and $(4,0)$ - this is the one shown in Fig. 2 -at $(3,0)$ and $(1,0)$, and at $(4,0)$ and $(2,0)$, having used periodicity on the last two. In general, that average contains $L$ terms on a $L \times L$ lattice;

(ii) the energy density $E$ which is defined as

$$
E=\frac{1}{2 L_{1} L_{2}} \frac{\partial \ln Z}{\partial \beta},
$$

and given by

$$
\begin{aligned}
E= & \frac{1}{2 L_{1} L_{2}} \sum_{x, \nu}\left\langle\cos \alpha(x) \cos \alpha\left(x+e_{\nu}\right)\right. \\
& \left.+\frac{I_{r(l)+1}(\beta \gamma(l))+I_{r(l)-1}(\beta \gamma(l))}{2 I_{r(l)}(\beta \gamma(l))} \gamma(l)\right\rangle,
\end{aligned}
$$

where $r(l)$ is defined in (11) and $\gamma(l)$ is $\gamma(l) \equiv \sin \alpha(x) \sin \alpha\left(x+e_{\nu}\right)$

(iii) the particle density. Since the number $N$ of particles is

$$
N=\frac{\partial \ln Z}{\partial \mu_{1}}=-\left\langle L_{1} q_{1}\right\rangle,
$$

we deduce that the particle density is

$$
n \equiv-\frac{1}{L_{2}}\left\langle q_{1}\right\rangle,
$$

or, equivalently, 

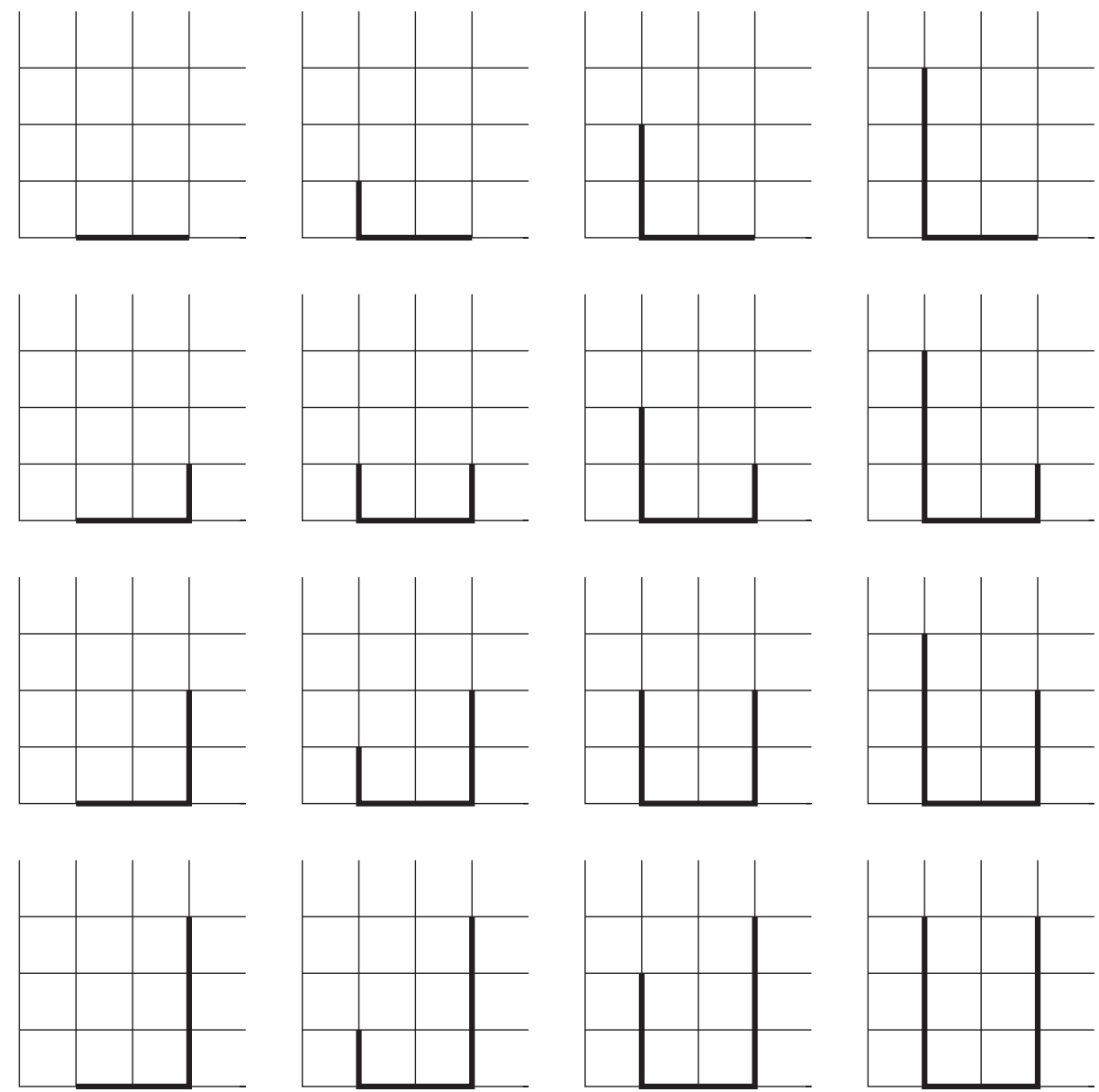

FIG. 2. Paths of a wall-wall correlation function at distance 2 on a $4 \times 4$ lattice. The walls are erected at sites $(2,0)$ and $(4,0)$.

$$
n \equiv-\frac{1}{L_{1}}\left\langle q_{2}\right\rangle \text {. }
$$

Both observables were measured for testing purposes as both (39) and (40) should provide the same result. The test was successfully passed.

\section{RESULTS}

The Monte Carlo simulations have been carried out on square lattices $L_{1}=L_{2} \equiv L$ with $L=20,30$, and 40 . $\mu_{2}$ was always set to zero and we will call $\mu \equiv \mu_{1}$. Having not too low acceptances is an indispensable condition to regard the results in every simulation run as valid. Therefore, since as shown in Fig. 1, our algorithm slowed down on large lattice sizes, we were compelled to employ $L \leq 40$. Figure 1 indeed exhibits the poor variability of $q_{1}$. We typically collected some $10^{7}$ measurements for each run.

Our strategy to construct the dual Boltzmann weight (10) allows to calculate every observable, including correlation functions. Such functions permit to extract the mass gap. This gap should coincide (i) with the value of the chemical potential $\mu$ at which nonzero particle numbers start off and (ii) with the value of $\mu$ at which the energy density detaches from its value at zero particle density. Therefore our procedure enables us to cross check the results for the mass gap.

Assuming the following theoretical form for the wallwall correlation function,

$$
\Gamma_{\mathrm{w}}^{(\mathrm{th})}(R)=A\left[e^{-R m}+e^{-(L-R) m}\right],
$$

we extracted the effective mass $m_{\mathrm{eff}}(R)$ as the value of the parameter $m$ at which the equality

$$
-\log \left[\frac{\Gamma_{\mathrm{w}}^{(\mathrm{MC})}(R+1)}{\Gamma_{\mathrm{w}}^{(\mathrm{MC})}(R)}\right]=-\log \left[\frac{\Gamma_{\mathrm{w}}^{(\mathrm{th})}(R+1)}{\Gamma_{\mathrm{w}}^{(\mathrm{th})}(R)}\right]
$$

holds, where $\Gamma_{\mathrm{w}}^{(\mathrm{MC})}(R)$ stands for the Monte Carlo determination of the wall-wall correlation. Here $m_{\mathrm{eff}}(R)$ is given in units of the inverse lattice spacing. We expect that $m_{\text {eff }}(R)$ exhibits a plateau for large enough $R$. The height of that plateau is the value of the mass gap resulting from our simulations. We call $m_{\mathrm{MC}}$ this height. In Fig. 3 we summarize our findings for two values of $\beta$ and $\mu$. This plot follows after measuring the correlation function on $2 \times 10^{7}$ configurations obtained in a $20 \times 20$ lattice. 


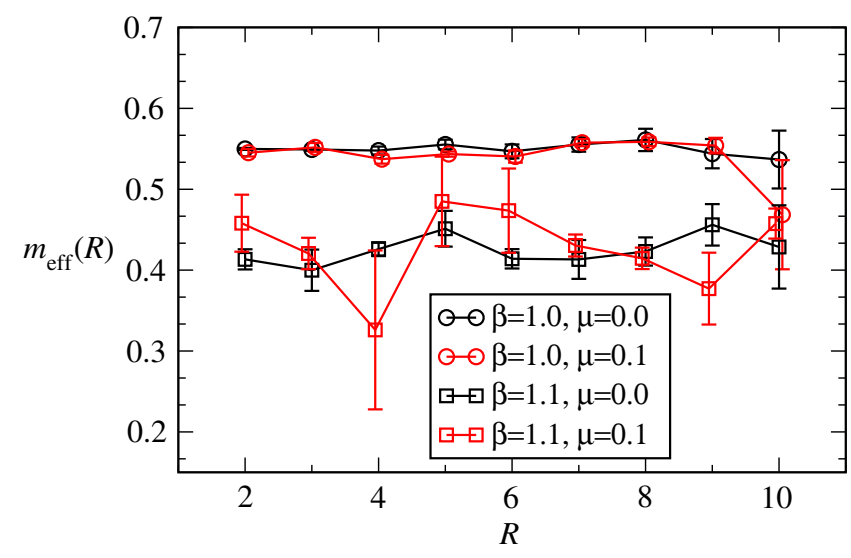

FIG. 3. Effective mass extracted from the correlator at different distances and values of $\mu$ for $\beta=1.0$ and $\beta=1.1$ on a $20 \times 20$ lattice. Lines are guides to the eye. The independence on $\mu$ is evident, as well as the presence of plateaux. All measurements have been done at integer values of $R$ (but some of them appear slightly shifted for readability).

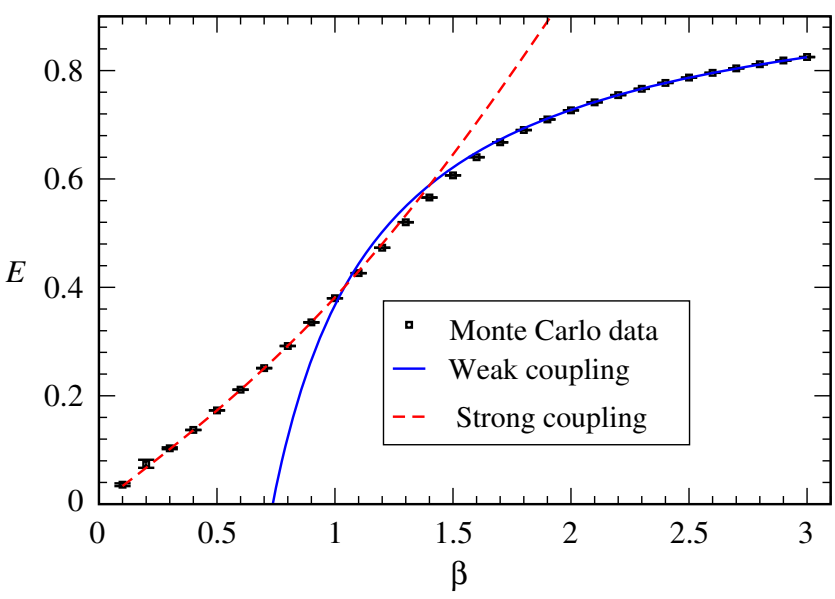

FIG. 4. Energy as a function of $\beta$ for $\mu=0$ on a $20 \times 20$ lattice. Monte Carlo data are represented by symbols $\square$. The result is in perfect agreement with the weak coupling and strong coupling expansions, represented respectively by continuous and dashed lines.

Similar results have been derived for other $\beta$ and $\mu$ (not shown). In all cases the expected independence on the chemical potential is apparent.

In Fig. 4 the energy density (37) for zero particle density is displayed as a function of $\beta$. Data in this figure are obtained again on a $L=20$ lattice. They exhibit a satisfactory agreement with the weak coupling expansion ${ }^{2}[26]$

$$
E=1-\frac{1}{2 \beta}-\frac{1}{16 \beta^{2}}-\frac{0.03851}{\beta^{3}}-\frac{0.03189}{\beta^{4}}+\cdots,
$$

\footnotetext{
${ }^{2}$ The coefficient of $1 / \beta^{4}$ includes the slight correction found in [25].
}

and with the strong coupling expansion [27]

$$
E=y+2 y^{3}+\frac{12}{5} y^{5}+\cdots, \quad y \equiv \frac{1}{\tanh \beta}-\frac{1}{\beta} .
$$

These agreements constitute further positive tests of our procedure.

Figure 5 shows the energy density $E$ as a function of $\beta$ and $\mu$ on a $20 \times 20$ lattice. As expected, the value of $\mu$ at which $E$ detaches from its value at $\mu=0$ is $\mu=m_{\mathrm{MC}}$. Even though we have used rather small lattice sizes, the coincidence between thresholds and effective mass $m_{\mathrm{MC}}$ is manifest because the energy density operator has negligible size effects.

Figure 6 shows the dependence of the particle density on $\mu$ for several $\beta$. Each point is the average of $10^{6}$ measurements. In principle also the thresholds in this plot should coincide with the plateaux in Fig. 3. This coincidence is not so manifest at $L=20$ because, contrary to the energy density, $n$ has strong size effects [21]. We have verified this assertion by repeating the study for $L=30$ and $L=40$ at $\beta=1.2$. Figure 7 blatantly exhibits that size dependence. While the threshold at $L=20$ is at about $\mu \approx 0.2$, at $L=$ 40 it is shifted to about $\mu \approx 0.3$. The point along the line for $\beta=1.2$ in Fig. 5 at which data detach from the value of the energy at zero density agrees reasonably well with the threshold in Fig. 7 for $L=40$.

Figure 7 also displays the consequences of the abovementioned slowing down. Indeed, although the three lines have been obtained with the same statistics, namely $2 \times 10^{7}$ measurements, all points on the line for $L=40$ and some of the points along the line for $L=30$ present larger error bars. They are due to long correlations among data. Such errors can be reduced only at the cost of increasing exorbitantly the statistics.

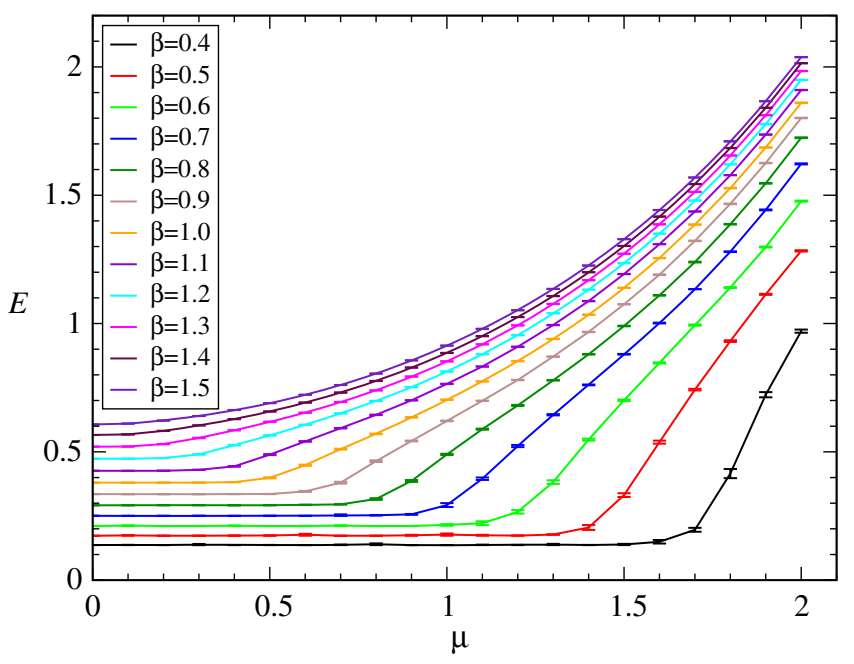

FIG. 5. Energy as a function of $\mu$ and $\beta$ on a $20 \times 20$ lattice. Lines are guides to the eye. The nontrivial dependence on $\mu$ starts only at $\mu=m_{\mathrm{MC}}$. 


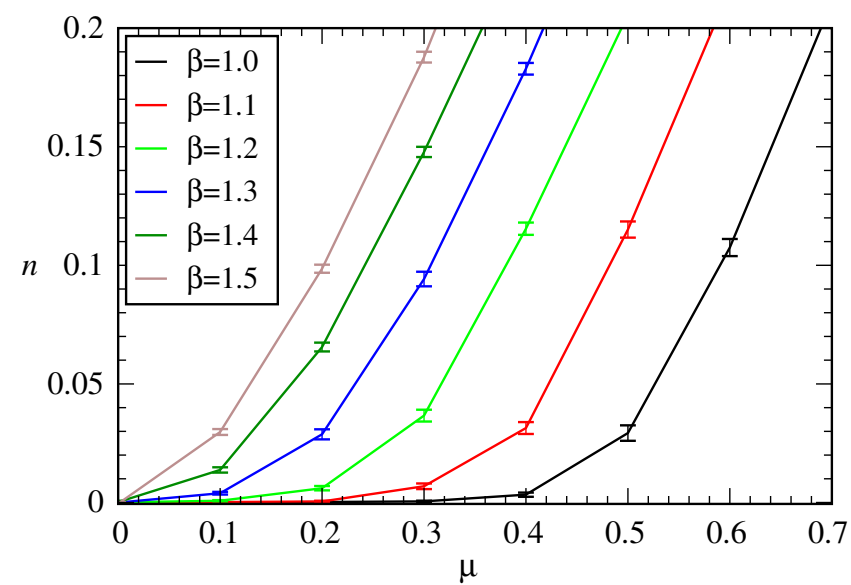

FIG. 6. Particle density as a function of $\mu$ and $\beta$ on a $20 \times 20$ lattice. Lines are guides to the eye. The threshold value for $\mu$ corresponds to the mass gap in units of the inverse lattice spacing and coincides with the thresholds shown in Fig. 5.

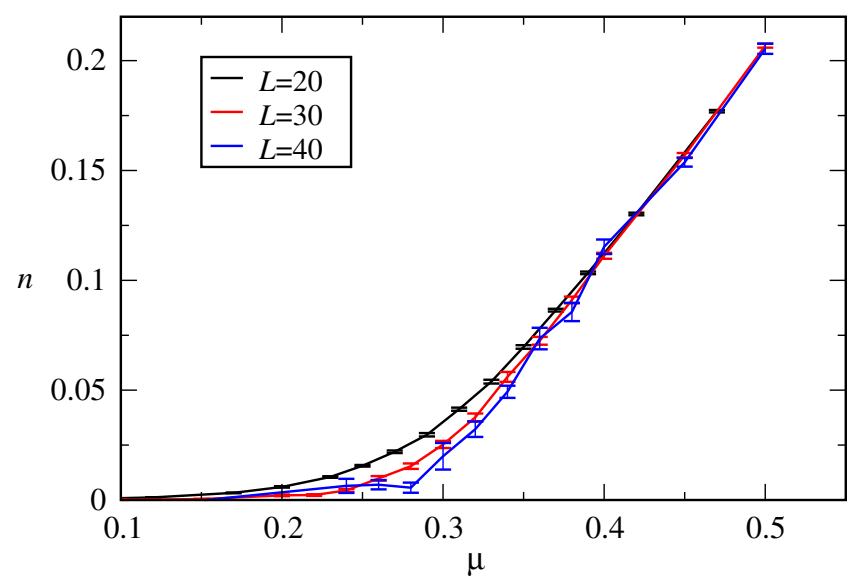

FIG. 7. Particle density as a function of $\mu$ at $\beta=1.2$ for $L=20$, 30,40 . Lines are guides to the eye. The threshold is evidently size dependent.

\section{DISCUSSION}

We have derived two different positive Boltzmann weights, (10) and (34), for simulating the 2D $O(3)$ nonlinear sigma model at nonzero chemical potential. These weights permit the evaluation of any observable average. The performances of both weights during Monte Carlo simulations are similar. For example in a wide region of $\beta-\mu$ the simulations run correctly, but low acceptances in the dynamical variables occur for both actions under certain values of $\beta, \mu$ and lattice sizes. This fact prevents the use of large lattices to avoid finite size effects, mainly at low, but non-zero, chemical potentials. Tempered Monte Carlo did not manage to speed up the simulation at those lattice sizes. Clearly all that results in strong slowing down effects on the simulation.
In turn, the above slowing down makes the values of the error bars to become large. This phenomenon is shown in Fig. 8 and Fig. 9 for the error bars of the energy and of the particle density. Such a growth raises the suspect that the sign problem may have not been completely beaten, even though the actions (10) and (34) derived in the paper are manifestly real. However, the fact that this slowing down looks more intense for low chemical potentials than for large ones (see Fig. 1) seems to indicate that its origin has nothing to do with the old sign problem (which worsens as $\mu$ increases) and is simply a spurious consequence of our procedure.

A detailed study of the type of functional growth of the error bars is hindered by the onset of the above-described slowing down at lattice sizes larger than 40 .

The above difficulties are absent on not too large lattice sizes. Thus, it is in those restrained sizes that we have

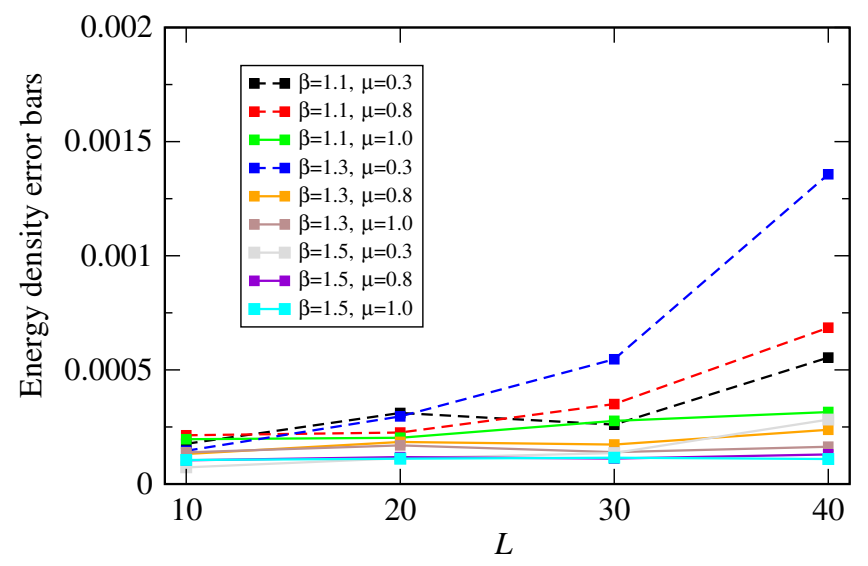

FIG. 8. Error bars for the energy density from a sample of $10^{7}$ data as a function of $L$. Lines are guides to the eye. Data exhibiting a steep growth of the error bars have been represented with dashed lines.

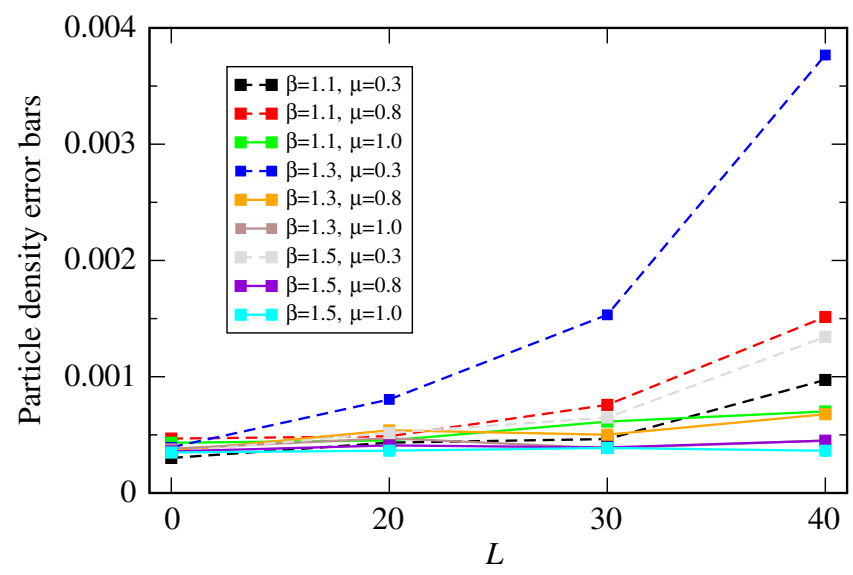

FIG. 9. Error bars for the particle density from a sample of $10^{7}$ data as a function of $L$. Lines are guides to the eye. Data exhibiting a marked growth of the error bars have been represented with dashed lines. 
measured all physically meaningful quantities, the sizes ranging from 10 to 40 . Specifically, we have extracted the correlation functions and from them the mass gap. These calculations have been confronted with the determination of the mass gap from the behaviour of the particle and energy densities as functions of $\mu$. The comparison has been successful. Also the energy density matches the weak and strong coupling expansions (where they should) for $\mu=0$.

It remains to investigate and solve the above slowing down and to perform a more accurate study of the model on larger lattices (which would enable us to address the question of an hypothetical BKT transition in $O(N)$ models at finite temperature and non-vanishing chemical potential). Appealing is also the possibility to extend the procedure described here to the $2 \mathrm{D} O(3)$ nonlinear sigma model with a topological $\theta$-term. In this case the sign problem is even more severe. In the past we derived a positive weight for the model at nonzero $\theta$ by mapping it to a certain dual version of the $S U(2)$ principal chiral model $[28,29]$. However, the worm algorithm applied for this dual model performed rather poorly during the Monte Carlo tests [29]. The approach described in this paper can be generalized to the model which includes the $\theta$-term. Whether or not one can construct the positive Boltzmann weight in this case or, at least, significantly reduce the sign problem remains to be verified.

\section{ACKNOWLEDGMENTS}

We gratefully acknowledge several illuminating discussions with Michele Caselle, Volodymyr Chelnokov and Marco Rossi. O. B. and B. A. also thank the Department of Physics of the University of Calabria for the warm hospitality during several stays. O. B. also thanks INFN (Fondo FAI) for financial support. B. A. and A. P. thank support from the INFN/NPQCD project. Numerical simulations have been run on the ReCaS Data Center of INFN-Cosenza.
[1] P. de Forcrand, Proc. Sci., Lattice2009 (2009) 010.

[2] V. Ayyar, S. Chandrasekharan, and J. Rantaharju, Phys. Rev. D 97, 054501 (2018).

[3] J. Bloch, J. Mahr, and S. Schmalzbauer, Proc. Sci., Lattice2015 (2016) 158.

[4] G. Aarts and K. Splittorff, J. High Energy Phys. 08 (2010) 017.

[5] Y. Tanizaki, Y. Hidaka, and T. Hayata, New J. Phys. 18, 033002 (2016).

[6] H. Fujii, S. Kamata, and Y. Kikukawa, J. High Energy Phys. 12 (2015) 125; 09 (2016) 172(E).

[7] A. Mukherjee and M. Cristoforetti, Phys. Rev. B 90, 035134 (2014).

[8] M. Cristoforetti, F. Di Renzo, A. Mukherjee, and L. Scorzato, Phys. Rev. D 88, 051501(R) (2013).

[9] A. Alexandru, G. Başar, P. F. Bedaque, G. W. Ridgway, and N.C. Warrington, Phys. Rev. D 95, 014502 (2017).

[10] P. Hasenfratz, M. Maggiore, and F. Niedermayer, Phys. Lett. B 245, 522 (1990).

[11] J.-K. Kim, Phys. Rev. D 50, 4663 (1994).

[12] B. Allés, G. Cella, M. Dilaver, and Y. Gündüç, Phys. Rev. D 59, 067703 (1999).

[13] A. M. Polyakov and A. A. Belavin, JETP Lett. 22, 245 (1975).

[14] V. A. Fateev, V. A. Kazakov, and P. B. Wiegmann, Nucl. Phys. B424, 505 (1994).

[15] G. V. Dunne and M. Unsal, Proc. Sci., Lattice 2015 (2016) 010 .
[16] F. Bruckmann, C. Gattringer, T. Kloiber, and T. Sulejmanpasic, Phys. Lett. B 749, 495 (2015).

[17] P. Meisinger and M. Ogilvie, Proc. Sci., Lattice 2013 (2014) 205.

[18] F. Bruckmann and T. Sulejmanpasic, Phys. Rev. D 90, 105010 (2014).

[19] F. Bruckmann, C. Gattringer, T. Kloiber, and T. Sulejmanpasic, Phys. Rev. D 94, 114503 (2016).

[20] F. Bruckmann, C. Gattringer, T. Kloiber, and T. Sulejmanpasic, Proc. Sci., Lattice 2016 (2016) 062.

[21] S. D. Katz, F. Niedermayer, D. Nógrádi, and Cs. Török, Phys. Rev. D 95, 054506 (2017).

[22] G. G. Batrouni and M. B. Halpern, Phys. Rev. D 30, 1775 (1984).

[23] O. Borisenko, V. Kushnir, and A. Velytsky, Phys. Rev. D 62 , 025013 (2000).

[24] N. A. Metropolis, A. W. Rosenbluth, M. N. Rosenbluth, A. H. Teller, and E. Teller, J. Chem. Phys. 21, 1087 (1953).

[25] B. Allés and M. Pepe, Nucl. Phys. B563, 213 (1999); B576, 658 (2000).

[26] B. Allés, A. Buonanno, and G. Cella, Nucl. Phys. B500, 513 (1997).

[27] B. Berg and M. Lüscher, Nucl. Phys. B190, 412 (1981).

[28] O. Borisenko, V. Kushnir, B. Allés, A. Papa, and C. Torrero, in Proceedings of International School-Seminar on New Physics and QCD at external conditions, Dnipropetrovsk (Dnipropetrovsk National University, Dnipro, 2013), p. 90.

[29] C. Torrero, O. Borisenko, V. Kushnir, B. Allés, and A. Papa, Proc. Sci., Lattice2013 (2014) 338. 